\title{
2 Ernährung, Flüssigkeits- und Volumentherapie - Besonderheiten im Management des adipösen Patienten
}

\author{
Thomas Bein
}

Ernährungsmanagement und Strategien der Flüssigkeitstherapie stellen in der Behandlung des kritisch kranken adipösen Patienten eine Besonderheit dar, da ein hohes Körpergewicht für Therapieüberlegungen ohne genaue Kenntnis der (patho)physiologischen Grundlagen zu Fehlentscheidungen führen kann: Es ist weder sinnvoll, übergewichtige Patienten während Intensivbehandlung durch stark reduzierte Kalorienzufuhr zum Abnehmen zu „zwingen“, noch sollte ein starkes Übergewicht zur übermäßigen Volumenzufuhr verleiten. Im Folgenden wird daher auf der Basis der Besonderheit metabolischer Veränderungen bei Adipositas sowie der aktuellen wissenschaftlichen Empfehlungen ein Vorschlag zum Ernährungsmanagement unterbreitet. Darüber hinaus finden sich - gestützt von grundsätzlichen Überlegungen zum Flüssigkeitsbedarf und zu speziellen Aspekten des Monitorings - einige Gedanken zur Strategie einer sinnvollen Volumenzufuhr für Patienten mit ausgeprägtem Übergewicht während operativer Eingriffe oder im Rahmen der Intensivbehandlung. Zu berücksichtigen ist allerdings, dass sowohl für Ernährungsbehandlung als auch für Flüssigkeitstherapie adipöser Patienten wenig gesicherte klinische Daten vorliegen und zum Teil wissenschaftliche Kontroversen über das „richtige“ Vorgehen geführt werden.

\subsection{Besonderheiten der metabolischen Reaktion auf Operation oder kritische Erkrankung bei Adipositas}

Menschen mit (starkem) Übergewicht weisen zum einen häufig verschiedene Begleiterkrankungen auf (Hypertonus, hypertensive Herzerkrankung, chronische Refluxstörung, Nierenfunktionsstörung, Gelenkerkrankungen), zum anderen liegen - mehr oder minder stark ausgeprägt - metabolische Störungen vor (diabetogene Stoffwechsellage, Fettstoffwechselstörung). Das metabolische Syndrom besteht aus einem Symptomenkomplex von vier Symptomen (abdominelle Fettleibigkeit, Dyslipidämie, Hypertonus, Insulinresistenz) und ist mit einer deutlich erhöhten Mortalität adipöser Patienten assoziiert. Die beschriebenen Begleiterkrankungen sowie metabolischen Störungen können im Rahmen von (ausgedehnten) Operationen oder anderen kritischen Erkrankungen, welche eine Intensivbehandlung erforderlich machen, erheblich aggravieren und den $\mathrm{Be}-$ handlungserfolg einschränken. Nach modernem Verständnis wird Adipositas als ein ,pro-inflammatorischer Zustand“ angesehen, in welchem die Schwelle für eine (überschießende) immunologische Reaktion auf Erkrankung oder Trauma nach unten verschoben ist (Port u. Apovian 2010). 
Vorbestehende Begleiterkrankungen und metabolische Störungen, welche bei Adipositas im gesunden Zustand im Stadium der Kompensation bestehen, treten im Rahmen einer schweren Erkrankung mit internistischer oder chirurgischer Intervention in den Vordergrund und bestimmen den Krankheitsverlauf.

Die genaue Kenntnis dieser pathologischen Veränderungen ist Voraussetzung für ein differenziertes Ernährungs- und Flüssigkeitsmanagement.

Patienten mit Übergewicht sind charakterisiert durch eine erhebliche Spannbreite bezüglich der krankheitsbedingten Veränderungen des Kohlenhydrat, Lipid- und Aminosäurenmetabolismus, welche erhebliche Variationen im Energiebedarf mit erhöhtem Proteinkatabolismus und tiefgreifende Änderungen von Immunfunktion und gastrointestinaler Leistung nach sich ziehen (Elamin 2005). Bei normalgewichtigen Patienten besteht die metabolische Antwort im Rahmen einer schweren Erkrankung in einem Anstieg des Protein- und Energiebedarfs, wobei eigene Reserven für eine Proteinsynthese eingesetzt werden. Als Mediatoren dieser Reaktion gelten Noradrenalin, Glukagon, Kortisol und Wachstumshormon, welche die Substratbewegungen zwischen den Organsystemen und den Geweben regulieren.

Im Gegensatz zu solch gut gesicherten Erkenntnissen bei Normalgewichtigen ist der metabolischhormonelle Effekt eines „Trauma“ bei Adipositas bisher nicht ausreichend untersucht. Es gibt allerdings Hinweise, dass - entgegen einer bisher geäußerten Vorstellung - die katabole Reaktion adipöser Patienten auf eine akute kritischer Erkrankung ebenso ausgeprägt, bzw. möglicherweise noch stärker ausfällt als im Vergleich zu Normalgewichtigen. Vermutlich ist - trotz der erhöhten Körpermasse - ein reduzierter Proteinspeicher bei adipösen Patienten hierfür verantwortlich. Es müssen daher überwiegend Lipide als Reaktion auf das „Postaggressionssyndrom“ freigesetzt werden, welches wiederum erhebliche (negative) Auswirkungen auf die Hormonkontrolle und die Blutzuckersteuerung nach sich zieht. Eine Verstärkung der (ohnehin meist vorbestehenden) Insulinresistenz mit weiterer Ausprägung der diabetogenen Stoffwechsellage ist häufig die Folge. Daher ist die Stress-induzierte Hyperglykämie ein häufiger Befund bei übergewichtigen Patienten während Intensivbehandlung oder im perioperativen Verlauf. Eine solche Hyperglykämie ist mit einer höheren Inzidenz an Infektionen und sonstigen Komplikationen vergesellschaftet.
Die katabole Reaktion von adipösen Patienten auf eine schwere Erkrankung („Postaggressionssyndrom“) ist mindestens ebenso ausgeprägt wie bei Normalgewichtigen. Der entscheidende Unterschied ist die Freisetzung von Fettsäuren, da die körpereigenen Proteinspeicher limitiert sind. In der Folge entwickelt sich häufig eine ausgeprägte Insulinresistenz mit Hyperglykämie, welche die Inzidenz von typischen Komplikationen (Infekte, Sepsis) erhöht.

\subsection{Einschätzung des Energiebedarfs bei Adipositas}

Die Einschätzung und Festlegung des Energiebedarfs von Patienten mit Übergewicht während Intensivbehandlung ist besonders schwierig, da - wie beschrieben - die metabolisch-hormonelle Reaktion auf eine Erkrankung im Gegensatz zu Normalgewichtigen extrem heterogen sein kann. In den letzten Jahren sind verschiedene Modelle zur Einschätzung des Energiebedarfs kritisch kranker Intensivpatienten entwickelt worden, die mehr oder minder gut für die klinische Praxis geeignet sind:

- Indirekte Kalorimetrie (,goldener Standard“)

- Harris-Benedict-Gleichung unter Einbeziehung von Alter und Größe

- Penn-State-Gleichung unter Einbeziehung von Atemminutenvolumen (bei beatmeten Patienten) und maximaler Körpertemperatur der letzten $24 \mathrm{~h}$

- American College of Chest Physicians unter Einbeziehung des ,idealen“ Körpergewichts

Gegenüber den vorgestellten Formeln gilt die individuelle Messung des Sauerstoffverbrauchs mittels indirekter Kalorimetrie als ,goldener Standard“. Allerdings gibt es derzeit kein in der klinischen Routine breit einsetzbares bettseitiges Verfahren zur individuellen Bestimmung des Energie-Ruheverbrauches („Resting energy expenditure “[REE]), aus dem der tägliche Energiebedarf („Total daily energy needs“[TEE]) durch Multiplikation mit einem „Stressfaktor“ ermittelt werden kann.

Bei der Anwendung solcher Formeln zur Ermittlung des Energiebedarfs adipöser Patienten besteht prinzipiell nach wie vor eine klinisch-wissenschaftliche Kontroverse, welches Gewicht zur Kalkulation verwendet werden soll: das tatsächliche oder das ideale Körpergewicht. In einigen Studien wurde unter Anwendung der indirekten Kalorimetrie nachgewiesen, dass die Verwendung des tatsächlichen Körpergewichts dem Energiebedarf deutlich besser 


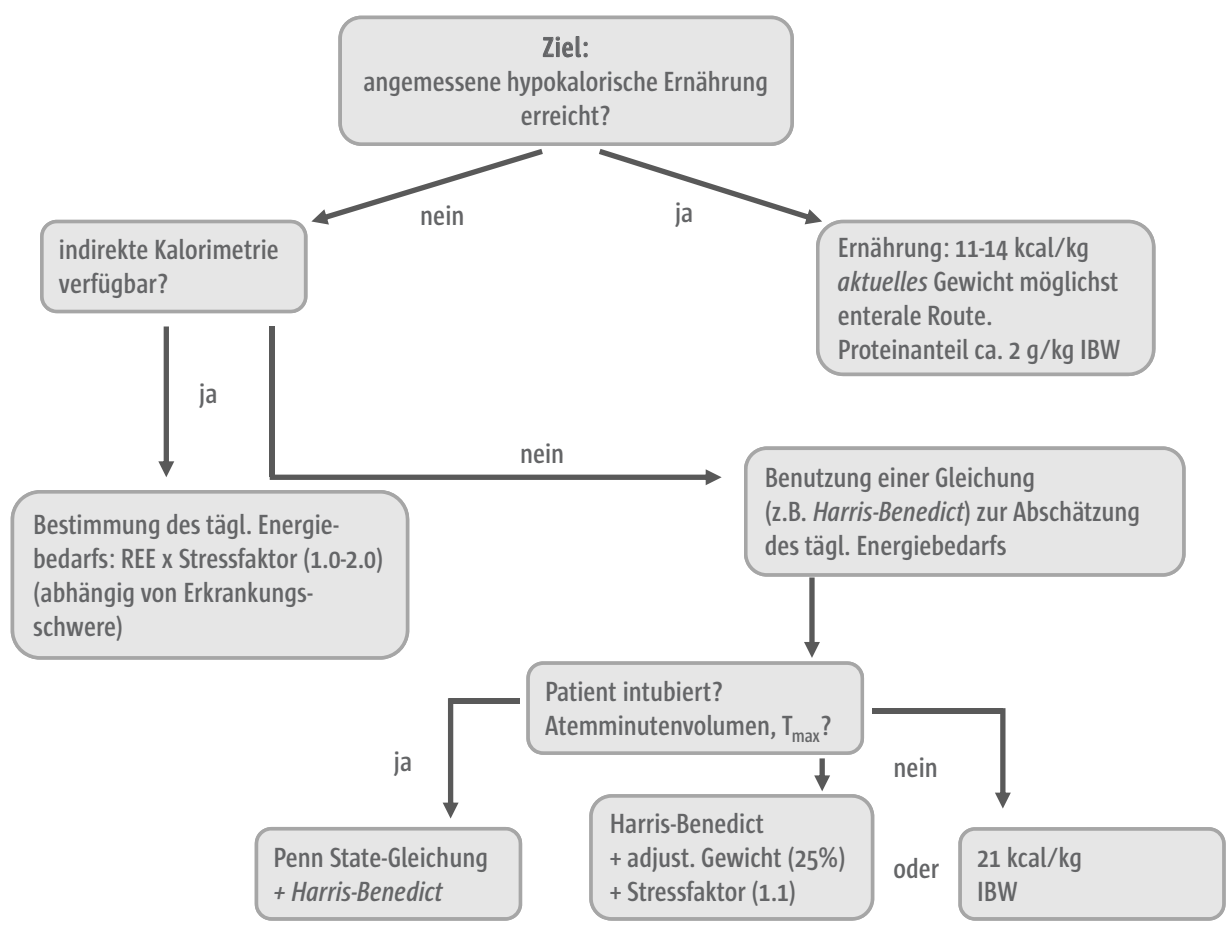

Abb. 2 Algorithmus zur Festlegung des täglichen Kalorienbedarfs kritisch kranker Patienten mit Adipositas (modifiziert nach Port u. Apovian 2010) REE = Ruheumsatz, IBW = ideales Körpergewicht, $T_{\max }=$ Körpertemperaturmaximum $/ 24$ h)

entspricht als die Verwendung des idealen Körpergewichts (s. Abb. 2).

Als „Golden Standard“ zur Bestimmung des Energiebedarfs adipöser Patienten während Intensivbehandlung gilt die indirekte Kalorimetrie, diese ist allerdings in der klinischen Routine wenig verbreitet.

Als am besten geeignete Formel erweist sich die Harris-Benedict-Gleichung unter Einbeziehung eines Stressfaktors.

Die Harris-Benedict-Formel lautet

(Gewicht = ideales Körpergewicht)

Energiebedarf Männer (kcal/24 h)

- $66,47+(13,75 \times$ Gewicht $[\mathrm{kg}])+(5 \times$ Körpergröße $[\mathrm{cm}])-$ $(6,75 \times$ Alter[Jahre]) x ,Stressfaktor“

Energiebedarf Frauen (kcal/24 h)

- $655,1+(9,56 \times$ Gewicht $[\mathrm{kg}])+(1,85 \times$ Körpergröße $[\mathrm{cm}])-$ (4,68 x Alter[Jahre]) x ,Stressfaktor"
Die Frage, ob und in welchem Ausmaß das für normalgewichtige Patienten akzeptierte Konzept der „hypokalorischen Ernährung “ auch für übergewichtige Patienten gilt, ist ebenfalls Gegenstand ausgedehnter wissenschaftlicher Untersuchungen gewesen (Dickerson 2005). In diesem Zusammenhang wird insbesondere die Gefahr der Verstärkung der hyperglykämen Stoffwechsellage durch inadäquate „Hyperalimentation“ betont. In der Zusammenschau der gegenwärtigen wissenschaftlichen Literatur gilt die hypokalorische Ernährung mit hohem Proteinanteil als wichtigste Maxime zur Ernährungstherapie adipöser Patienten. Hierbei gilt es, die mit einer Überernährung assoziierten Komplikationen, insbesondere die Hyperglykämie, zu vermeiden. Darüber hinaus ist die Reduktion des ProteinKatabolismus ein bedeutendes Ziel. Allerdings sollte das Konzept der „hypokalorischen Ernährung“ unter keinen Umständen als „Fastendiät“ fehlgedeutet werden, da - wie oben erwähnt - ein Unterschreiten einer hypokalorischen Schwelle unweigerlich die Katabolismus-Spirale verstärkt und die Überlebenschance verschlechtert. 
Ein mittels angewendeter Formel (z.B. Harris-Benedict) oder anhand des tatsächlichen Körpergewichts (s. Abb. 2) gewähltes angemessenes hypokalorisches Ernährungskonzept mit hohem Proteinanteil ist das beste Vorgehen für adipöse Patienten. Sowohl die Hyperalimentation (Hyperglykämie!), als auch eine „Fastendiät“ (Verstärkung des Proteinkatabolismus!) ziehen eine erhöhte Komplikations- und Mortalitätsrate nach sich.

Die Intensivbehandlung ist kein geeigneter Zeitpunkt, einen adipösen Patienten zum „Abnehmen“ zu nötigen. Im Gegenteil: Ein „Fastenversuch“ erhöht die Sterblichkeit!

\subsection{Auswahl und Zusammenstellung der Substrate}

Im ersten Teil dieses Kapitels wurden die Unterschiede und (teils unabsehbaren) typischen Reaktionen des adipösen Organismus auf operativen Stress, Trauma oder innerer Erkrankung dargelegt: Der Fettleibige verfügt über einen geringeren Proteinspeicher als der Normalgewichtige. Solche Proteinspeicher werden im Rahmen des „Postaggressionssyndroms " eingesetzt, um den Proteinkatabolismus abzufangen. Bei fehlendem Proteinspeicher kommt es verstärkt zu Proteinabbau. Das bedeutet für den Adipösen, dass bei nicht-angemessener Ernährung vermehrt Muskelmasse abgebaut wird - eine fatale Entwicklung. Wie erwähnt, empfiehlt sich daher eine Ernährung mit hohem Proteinanteil bei Adipositas.

Um einem verstärkten Proteinkatabolismus = Abbau von Muskelmasse vorzubeugen, empfiehlt sich für adipöse Patienten die Zufuhr einer Ernährung mit hohem Proteinanteil.

Die gemeinsamen Leitlinien der Society of Critical Care Medicine (SCCM) und der American Society for Parenteral and Enteral Nutrition (ASPEN) stellen daher folgende Empfehlungen für den Proteingehalt der Ernährung kritisch kranker adipöser Patienten vor (McClave et al. 2009):

- Body-Mass-Index 30-40

- Gesamtkalorien 11-14 kcal/kg aktuelles Gewicht oder 22-25 kcal $/ \mathrm{kg}$ ideales Gewicht

- Proteine $\geq 2, \mathrm{og} / \mathrm{kg}$ ideales Körpergewicht
- Body-Mass-Index $\geq 40$

- Gesamtkalorien 11-14 kcal/kg aktuelles Gewicht oder 22-25 kcal/kg ideales Gewicht

v Proteine $\geq 2,5 \mathrm{~g} / \mathrm{kg}$ ideales Körpergewicht

Wie bei normalgewichtigen Patienten auch, sollte als Ernährungszugang frühzeitig die enterale Route via Magensonde gewählt werden. Allerdings besteht bei adipösen Patienten häufig eine Refluxerkrankung, sodass eine niedrige Dosierung (20-30 ml Sondenkost/h) häufig zunächst nicht steigerbar ist und auch nicht gesteigert werden sollte. Es empfiehlt sich auf jeden Fall, auch bei dieser Patientengruppe das Konzept der „minimalen enteralen Ernährung“ konsequent fortzuführen und zum Erreichen der errechneten täglichen Kalorienzufuhr die parenterale Ernährung dazu zu kombinieren.

\subsection{Flüssigkeitsmanagement}

Im Vergleich zum Ernährungskonzept liegen für spezielle Aspekte des Flüssigkeitsmanagements bei adipösen Patienten viel weniger gesicherte Studiendaten oder Empfehlungen vor. Die wenigen publizierten Studien sind im perioperativen Setting bariatrischer Operationen durchgeführt worden. Hier wurde der Frage nachgegangen, ob ein „liberales“ Flüssigkeitsmanagement mit ausgeprägter Gabe kristalloider Flüssigkeit (40 ml/kg aktuelles Körpergewicht) bezüglich der Vermeidung von postoperativen Nierenfunktiosstörungen oder der Entwicklung einer Rhabdomyolyse einem restriktiven Management (15 ml/ $\mathrm{kg}$ ) überlegen sei (Wool et al. 2010). Die Patienten mit "liberaler" Flüssigkeitszufuhr hatten eine signifikant höhere Urinproduktion während der Operation, im Aufwachraum und am 1. postoperativen Tag. Darüber hinaus zeigte sich ein Trend zu einem niedrigeren Serum-Kreatinin-Wert. Allerdings waren die Gruppen nicht unterschiedlich hinsichtlich der postoperativen Erhöhung der Creatin-Kinase-Serum-Werte (CK) oder der Entwicklung einer Rhabdomyolyse. Die Autoren empfehlen daher nicht ausdrücklich ein liberales Flüssigkeitsmanagement für das perioperative Setting bariatrischer Operationen.

Welche Parameter sind zum Monitoring und somit zur Steuerung der Volumenzufuhr bei Patienten mit massiver Adipositas geeignet? Dieser Frage ist in einer anderen Studie mit ähnlichem Setting nachgegangen worden (Jain u. Dutta 2010). Im perioperativen Verlauf bariatrischer Operationen bei $50 \mathrm{~Pa}-$ tienten mit massiver Adipositas wurde der funktionelle Parameter „Schlagvolumenvariation“(SVV), er- 
fasst mittels Pulskonturanalyse mit den „traditionellen" Parametern (Herzfrequenz, nicht-invasive Blutdruckmessung, zentraler Venendruck), zur Erfassung des Volumenstatus verglichen. Es zeigte sich, dass der Parameter Schlagvolumenvariation besser geeignet war, den Volumenbedarf zu ermitteln und dass die herkömmlichen Parameter Blutdruck und zentraler Venendruck eher zu einer Überinfusion verleiten. Insgesamt schließen die Autoren aus ihren Befunden, dass übergewichtige Patienten im Rahmen von Operationen nicht mehr Volumen benötigen als Normalgewichtige. Es wird daher davor gewarnt, übergewichtigen Patienten mehr und zuviel Volumen zuzuführen und somit häufig bestehende Begleiterkrankungen (z.B. hypertensive Herzerkrankung) zu verstärken.

Nach den wenigen, derzeit zur Verfügung stehenden, Studienergebnissen und der physiologischen Rationale besteht kein Anlass, übergewichtigen Patienten mehr Volumen zu verabreichen als normalgewichtigen Patienten.

Die Hypothese „Wer viel wiegt, der muss auch viel Flüssigkeit bekommen" ist nach gegenwärtigem Erkenntnisstand irrig! Zu viel Volumen kann wohl eher im Sinne einer Begünstigung der kardialen Belastung schaden. Dies gilt in jedem Fall für den perioperativen Verlauf, wahrscheinlich aber auch für die
Intensivbehandlung, obwohl hierfür keine Untersuchungen vorliegen.

Als Monitoring eignet sich offensichtlich besonders die Analyse der Schlagvolumenvariation, da nicht-invasive Blutdruckmessung und zentraler Venendruck nach vorliegenden Studienergebnissen eher zur ausgiebigen Volumengabe (ver)führen.

\section{Literatur}

Dickerson RN (2005) Hypocaloric feeding of obese patients in the intensive care unit. Curr Opin Clin Nutr Metab Care 8, 189-196

Elamin E (2005) Nutritional Care of the obese intensive care unit patient. Curr Opin Crit Care 11, 300-303

Jain AK, Dutta A (2010) Stroke volume variation as a guide to fluid administration in morbidly obese patients undergoing laparoscopic bariatric surgery. Obes Surg 20, 709-715

McClave SA, Martindale RG, Vanek VW, McCarthy M, Roberts P, Taylor B, Ochoa JB, Napolitano L, Cresci G; A.S.P.E.N. Board of Directors; American College of Critical Care Medicine; Society of Critical Care Medicine. (2009) Guidelines for the provision and assessment of nutrition support therapy in the adult critically ill patient. Parenter Enteral Nutr 33, 277-316

Port AM, Apovian C (2010) Metabolic support of the obese intensive care unit patient: a current perspective. Curr Opin Clin Nutr Metab Care 13, 184-191

Wool DB, Lemmens HJM, Brodsky JB, Solomon H, Chong KP, Morton JM (2010) Intraoperative fluid replacement and postoperative creatine phosphokinase levels in laparoscopic bariatric patients. Obes Surg 20, 698-701

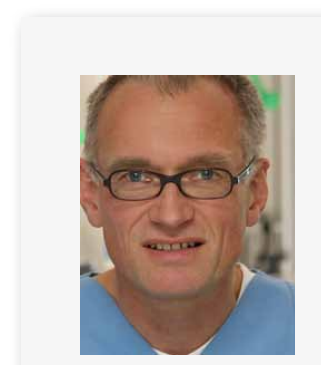

\section{Prof. Dr. med. Thomas Bein}

Studium der Humanmedizin in Marburg und München. Ausbildung zum Facharzt für Anästhesiologie am Universitätsklinikum der Ludwig-Maximilians-Universität, Innenstadt und Großhadern. Oberarzt der Anästhesiologischen Intensivstation Klinikum Großhadern. Wechsel an die neu gegründete Universitätsklinik Regensburg und Aufbau der Intensivstation. 1995 Forschungsaufenthalt in Uppsala, Schweden.

1997 Habilitation. Seit 2002 Professur und Leitung der Operativen Intensivstation. Forschungsschwerpunkte: Behandlungsstrategien beim akuten Lungenversagen, extrakorporaler Gasaustausch. 